\title{
Studies of mildly relativistic magnetized perpendicular shocks with kinetic simulations
}

\author{
Arianna Ligorini*, Jacek Niemiec, \\ Institute of Nuclear Physics - Polish Academy of Science, ul. Radzikowskiego 152, 31-342 \\ Kraków, Poland \\ E-mail: arianna.ligorini@ifj.edu.pl

\section{Pohl} \\ Institute of Physics and Astronomy, University of Potsdam, Karl-Liebknecht-Strasse 24/25, \\ 14476 Potsdam, Germany \\ DESY, Platanenallee 6, 15738 Zeuthen, Germany
}

\begin{abstract}
To provide an interpretation of X-ray and gamma-ray emission spectra of jets in Active Galactic Nuclei (AGN) a detailed knowledge of particle acceleration at dissipation sites is required. In the case of blazars, the dissipation of jet energy may involve mildly relativistic magnetized internal shocks with Lorentz factor of $\gamma \leq 2$. Of special interest for the emission modeling is the level of kinetic energy exchange between protons and pre-heated electrons. While studies of ultrarelativistic collisionless shocks demonstrate strong electron-ion coupling, mediated by the electron maser instability in the shock precursor, the processes of shock formation, electromagnetic turbulence production, and particle acceleration in the mildly relativistic regime are poorly known. Here we present preliminary results of fully kinetic particle-in-cell simulation studies of mildly relativistic superluminal shocks in strongly magnetized plasma. The main focus is on ion-to-electron energy transfer processes and the effects of the electron-positron pair content.
\end{abstract}

35th International Cosmic Ray Conference - ICRC2017 -

10-20 July, 2017

Bexco, Busan, Korea

* Speaker. 


\section{Introduction}

Relativistic quasi-perpendicular (superluminal) shocks are thought to be responsible for emission in AGN jets and gamma-ray bursts. Such shocks propagating in the magnetized plasma and in the regime of ultra-relativistic shock velocities should be inefficient particle accelerators unless, e.g., some pre-heating mechanism causes electrons to acquire gyroradii larger than the thickness of the shock, so that they can experience multiple shock crossings and become energized before being taken away downstream. The same mechanism can lead to efficient ion-to-electron energy transfer resulting in electron heating up to equipartition with ions. Such strong electron-ion coupling in the relativistic regime has been proposed to explain leptonic emission models for blazars [1].

Superluminal shocks are mediated by magnetic reflection of the incoming flow off the shockcompressed magnetic field [2,3]. Under magnetized conditions, coherent gyration of the incoming particles at the shock front may trigger the so-called synchrotron maser instability (SMI) $[4,5]$ : when a ring of particles gyrating in the shock transition zone breaks up in bunches of charge, these bunches radiate a coherent train of transverse electromagnetic waves of the extraordinary mode (X-mode) which can escape towards the upstream region $[6,7,8]$. In electron-ion plasma, incoming electrons can then experience transverse oscillations in the strong field of these precursor waves and their guiding-center velocity decreases. Since ions are less affected by the waves, due to their heavier mass, they proceed at roughly their initial velocity. The resulting difference in bulk velocities between the two species generates a longitudinal electric field (wakefield) [5]. This field can cause electrons to be boosted toward the shock and accelerated [9]. At the same time, ions are still not appreciably affected. As a result, energy equipartition between electrons and ions may be achieved even before the flow arrives at the shock front.

Precursor waves generation at relativistic magnetized shocks has been demonstrated in a number of studies using one-dimensional (1D) particle-in-cell (PIC) simulations [7, 6, 9, 10, 11]. Such shocks have recently been also studied with two-dimensional (2D) simulations for the case of a pure pair plasmas [3,12], pure electron-ion plasmas [13,14] and also electron-ion plasmas with a positron content [14]. While most of 2D studies report precursor waves, the efficiency of their emission is still debated [12]. The latter has direct consequences on the level of the electron-ion coupling and the ability of superluminal shocks to produce non-thermal electrons. However, most of these works treat ultra-relativistic shocks with Lorentz factors $\gamma \gtrsim 10[13,14]$. The mildly relativistic regime, $\gamma \lesssim 2$, is instead poorly known. Here we study mildly relativistic highly magnetized superluminal shocks in pure electron-ion plasmas to address the shock structure and the electron-proton coupling. Preliminary results will be presented.

\section{Simulation setup}

We use first-principles PIC simulations that offer a self-consistent ab-initio model of collisionless plasma. The simulations are based on advanced MPI-based parallel relativistic codes using finite-difference algorithms to follow individual macro-particle trajectories and solve Maxwell equations for the electromagnetic fields on a spatial grid. Here we use a modified version of the TRISTAN code [15]. The simulation setup is shown in Fig. 1. We inject two equal-density and cold electron-ion beams counter-streaming along the x-direction. The beams carry a large-scale 
magnetic field that is set to be perpendicular to the simulation plane (x-y). Corresponding motional electric field $\mathbf{E}=-\mathbf{v} \times \mathbf{B}_{\mathbf{0}}$ is also initialized in both plasmas and lies in the simulation plane. Upon collision of the two beams a system of two oppositely-propagating shocks separated by a contact discontinuity $(\mathrm{CD})$ is formed. We probe a mildly relativistic regime and set the beams Lorentz factors $\gamma_{0}$ so that the resulting shock moves with $\gamma_{s h}=2$. Furthermore, we assume a total plasma magnetization $\sigma=0.1$, as expected in AGN shocks, with $\sigma$ defined as $\sigma=B_{0}^{2} /\left(\mu_{0} N_{i}\left(m_{e}+m_{i}\right) \gamma_{0} c^{2}\right)$, where $c$ is the speed of light, $\mu_{0}$ is the permeability of free space and the upstream quantities: magnetic field strength, $B_{0}$, and ion density, $N_{i}$, are given in the simulation (shock downstream) frame [7]. With such definition, $1 / \sigma=1 / \sigma_{e}+1 / \sigma_{i}$, where $\sigma_{e}$ and $\sigma_{i}$ are electron and ion magnetizations, respectively. We assume a reduced ion-to-electron mass ratio of $m_{i} / m_{e}=50$. The unit of length used here is the ion skin depth, $\lambda_{s i}=c / \omega_{p i} \sim 106$ cells, with $\omega_{p i}$ - the ion plasma frequency. Corresponding electron skindepth, defining the spatial resolution, is $\lambda_{s e}=15$ cells. Time is expressed in units of the ion cyclotron frequency $\Omega_{c i}$. As we collide two symmetric plasma beams, in the following we present results only for the shock moving to the right from the CD.

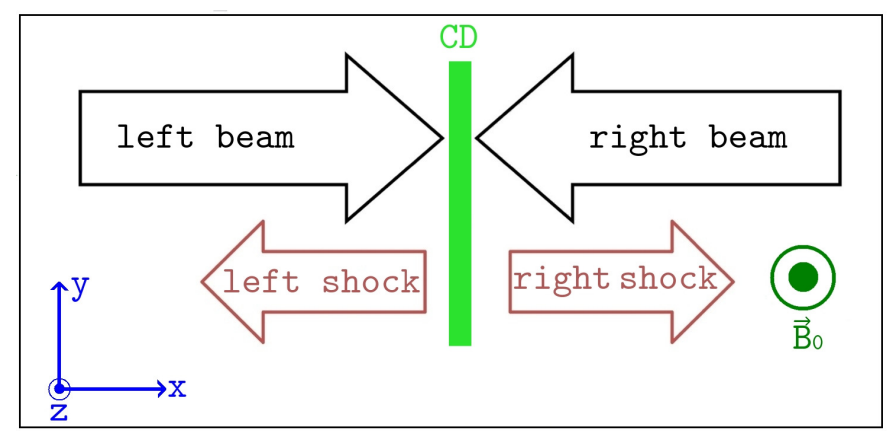

Figure 1: Illustration of our 2D simulation setup.

\section{Results}

Our study is based on 2D3V simulations of perpendicular shocks. However, to compare with earlier 1D works we also present results of $1 \mathrm{D} 3 \mathrm{~V}$ modeling. The latter uses a 2D setup and sets 5 grid cells in the $y$-direction. To have good statistics we use 100 particles per cell per species in this run.

The shock structure in the $1 \mathrm{D}$ run at time $t=139.8 \Omega_{c i}^{-1}$ is shown in Figure 2. The shock front is located at $\left(x-x_{C D}\right) / \lambda_{s i} \approx 100$ from the $\mathrm{CD}$, where a density increase by a factor of $\sim 3$ can be identified, as expected from the shock jump conditions. Upstream of the shock, largeamplitude short-wave fluctuations in $B_{z}$ magnetic field can be observed (Fig. 2c). They move with velocity close to $c$ and their wavelength is $\lambda_{B z} \sim 0.5 \lambda_{s i}{ }^{1}$ The waves of the same wavelength and

\footnotetext{
${ }^{1}$ In the analysis of the precursor waves in 1D and 2D simulations we consider only the "central part" of these waves, excluding from our analysis the fluctuations near the tip of the precursor waves, as they may be contaminated by initial conditions.
} 


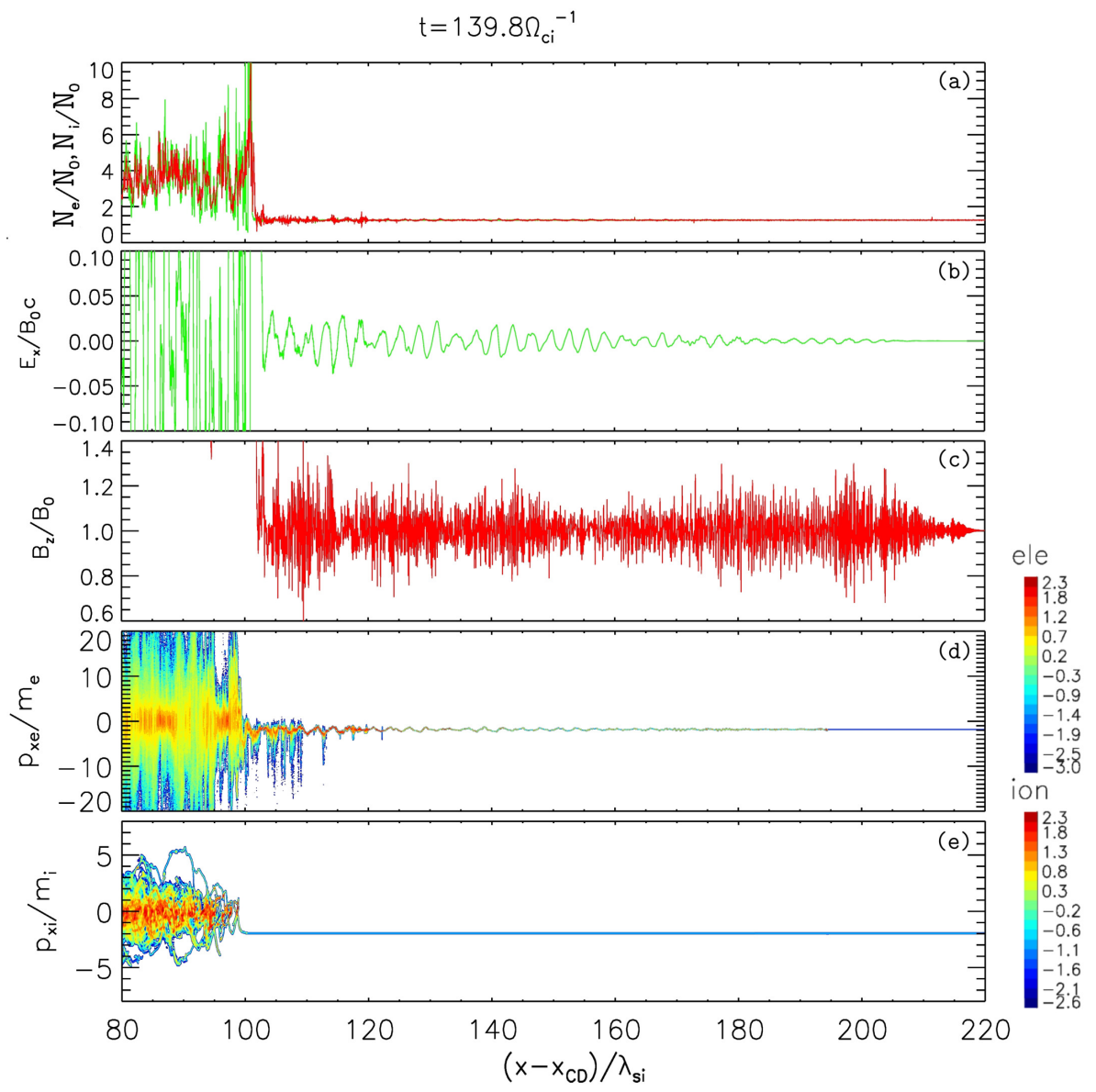

Figure 2: Shock structure in $1 \mathrm{D}$ simulation at time $t=139.8 \Omega_{c i}^{-1}$. As a function of the distance from the $\mathrm{CD} x-x_{C D}\left[\lambda_{s i}\right]$ we plot: (a) electron and ion number density, normalized to their upstream value, (b) perpendicular magnetic field, $B_{z}$, and (c) longitudinal electric field, $E_{x}$, both normalized to the upstream magnetic field, $B_{0}$, and the longitudinal phase space for (d) electrons and (e) ions.

amplitude are seen in $E_{y}$ electric field, which means that they are linearly polarized along the ambient magnetic field ( $z$-direction). As this is a signature of the X-mode wave, we identify these fluctuations with the precursor waves generated via SMI. As noted, in the electron-ion plasma the precursor waves should induce a wakefield. In fact, upstream oscillations are clearly visible in $E_{x}$ (Fig. 2b). Their wavelength, $\lambda_{E x} \sim 3 \lambda_{s i}$, is in agreement with theoretical estimates for wakefield originating through the Forward Raman Scattering [9], that predict $\lambda \sim 3.55 \lambda_{s i}$ for the value of $\gamma$ used in our simulations.

As expected in a superluminal shock, no particle can be reflected from the shock and propagate back upstream. Correspondingly, we do not find particles with positive $x$-momentum in phase-space distributions shown in Figures 2d-e. On the other hand it is possible to detect in ion phase-space a ring-like feature at the shock, which could be a signature of ions gyrating around compressed magnetic field in the shock. Similarly, in electron phase-space faint oscillations downstream of the shock can be spotted. The electron longitudinal phase-space is modulated upstream 


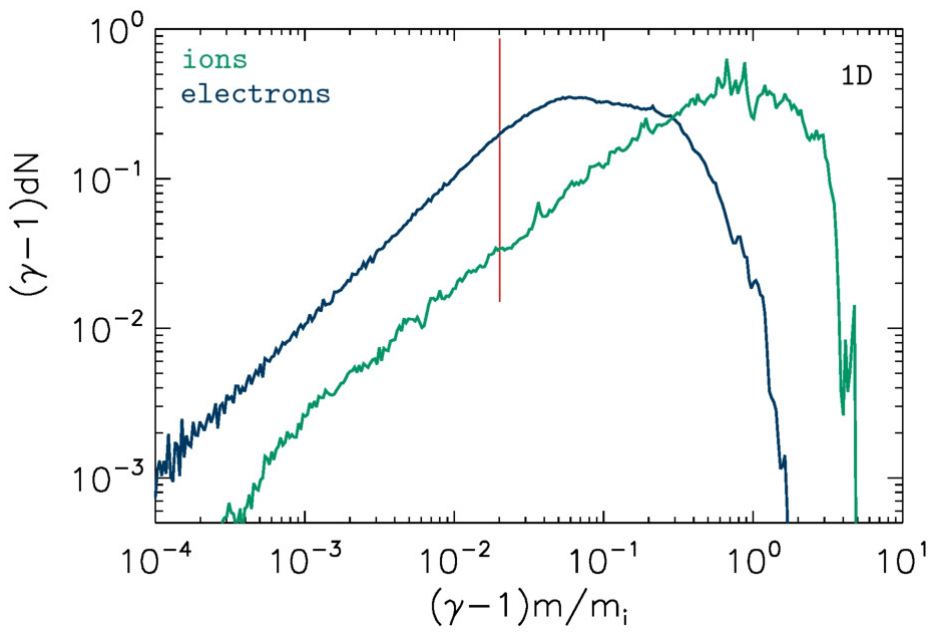

Figure 3: Particle energy spectra in the $1 \mathrm{D}$ run at time $t=139.8 \Omega_{c i}^{-1}$ in the downstream region, $40 \lambda_{s i}$ from the shock position, and integrated on a width of $20 \lambda_{s i}$. Red line marks the location at $(\gamma-1) m_{e} / m_{i}$ expected for the peak of electron distribution for the thermalized upstream plasma without ion-to-electron energy transfer.

with the wavelength of the wakefield $E_{x}$ oscillations. This demonstrates that the incoming plasma is perturbed by the precursor waves. Due to their lighter mass, electrons are more affected by this process, and indeed they oscillate in the field of the precursor waves, while ions are not disturbed. Electron interactions with wakefields cause them to be boosted towards the shock (i.e., in negative $x$-momentum), as it can be seen in Fig. $2 \mathrm{~d}$ in the upstream region close to the shock, at $\left(x-x_{C D}\right) / \lambda_{s i} \approx 100-120$, where the amplitude of the $E_{x}$ oscillations is the largest. The precursor waves can thus facilitate an energy transfer from ions to electrons.

Figure 3 shows electron and ion spectra downstream of the shock for the 1D run. Ions are isotropized around their initial energy and form a relativistic Maxwellian distribution. Electrons are also thermalized close to their initial energy (red vertical line in Fig. 3) and are only slightly heated in bulk. Some asymmetry in the electron distribution at high energies might arise from acceleration upstream in the wakefield electric field.

The shock structure in 2D run is shown in Figure 4. This simulation has been performed using a transverse size of the computational box with $L_{y}=584$ cells, corresponding to $L_{y} \sim 5 \lambda_{s i}$. The simulation ran for $t=139.8 \Omega_{c i}^{-1}$ and involved the particle density of 10 particles per cell per species; all other parameters are the same is in the $1 \mathrm{D}$ run. The shock is again located at $\left(x-x_{C D}\right) / \lambda_{s i} \approx 103$, consistent with 1D run. Other characteristics of the 2D shock structure are similar to that observed in the 1D run - precursor waves are emitted upstream and induce wakefield oscillations that cause one-sided (in $p_{x}$ ) electron acceleration. The wavelengths of the precursor waves and electrostatic oscillations in $E_{x}$ are the same as that obtained in 1D simulation. However, the amplitude of both $\mathrm{X}$-mode waves and wakefield is an order of amplitude smaller in our 2D run, than in the 1D case.

Despite suppression of the wave amplitudes in the precursor of the 2D shock with respect to results obtained in 1D simulation, the level of electron energization by the wakefield oscillations seems to be similar in both runs (Fig. 4d). Correspondingly, particle spectra downstream of the 
2D shock demonstrate similar level of coupling between electrons and ions (Fig. 5). A proper interpretation of this result requires further scrutiny.

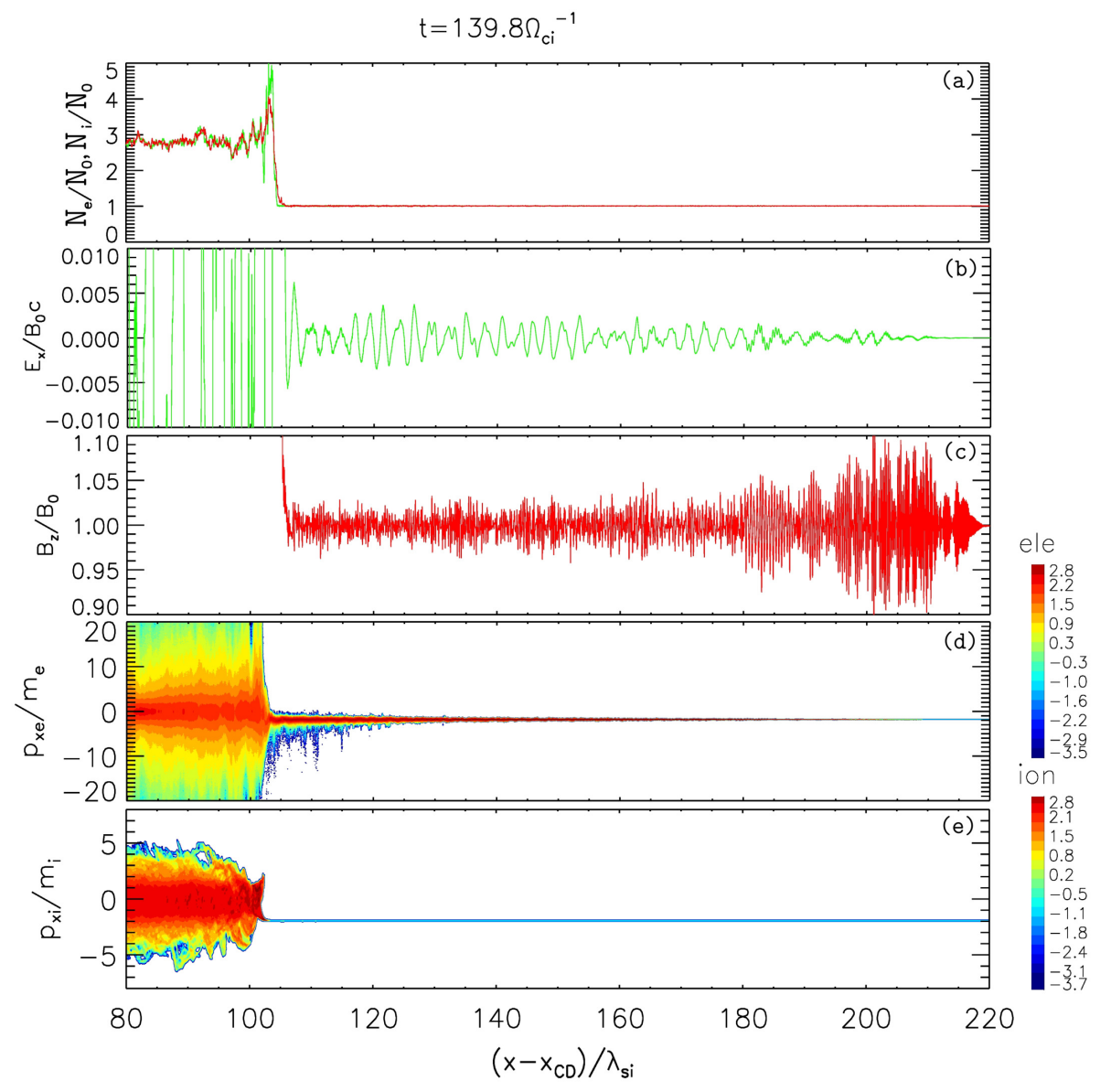

Figure 4: Shock structure in 2D simulation at time $t=139.8 \Omega_{c i}^{-1}$ (compare with Fig. 2).

\section{Discussion and conclusions}

Preliminary results of PIC simulations of a poorly explored regime of mildly relativistic magnetized shocks in ion-electron plasmas have been presented. We based our discussion on 1D3V and $2 \mathrm{D} 3 \mathrm{~V}$ simulations that show consistent evidence for the presence of the synchrotron maser instability that triggers electromagnetic waves propagating towards the shock upstream and inducing electrostatic wakefield oscillations. The latter cause oscillations in the upstream phase-space distribution of the electrons and one-sided electron heating close to the shock, where wakefields have the largest amplitudes. Particle-wave interactions in the shock precursor lead to plasma thermalization and limited ion-to-electron energy transfer.

These features are consistent with results obtained by [13] in long-term simulations of shocks with Lorentz factor down to $\gamma=3$, who noted suppression of the precursor wave amplitudes and electron heating with decreasing $\gamma$. However, our preliminary results show relatively early-stage 


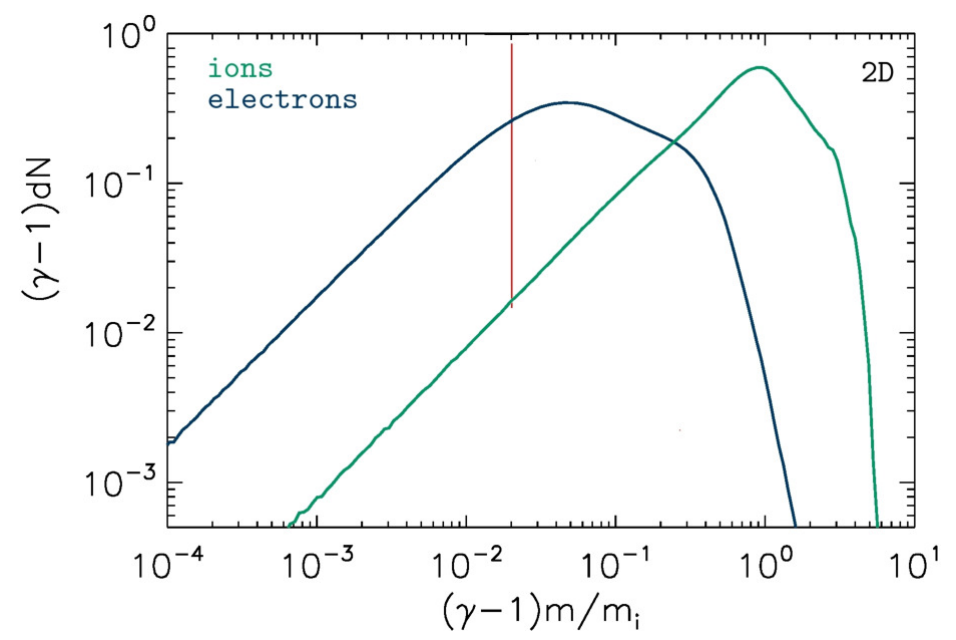

Figure 5: Particle energy spectra downstream of the shock in the $2 \mathrm{D}$ run at $t=139.8 \Omega_{c i}^{-1}$ (see Fig. 3).

evolution of the system. Moreover, the efficiency of the precursor wave emission observed in our simulations, as well as in simulations presented in [13], may be influenced by the choice of relatively low spatial resolution (and particle-per-cell number in the 2D run), as noted recently in [12]. Finally, a careful analysis of the effects of reduced ion-to-electron mass ratios needs to be performed.

Some insight on the latter may be provided by noting that for a given value of total plasma

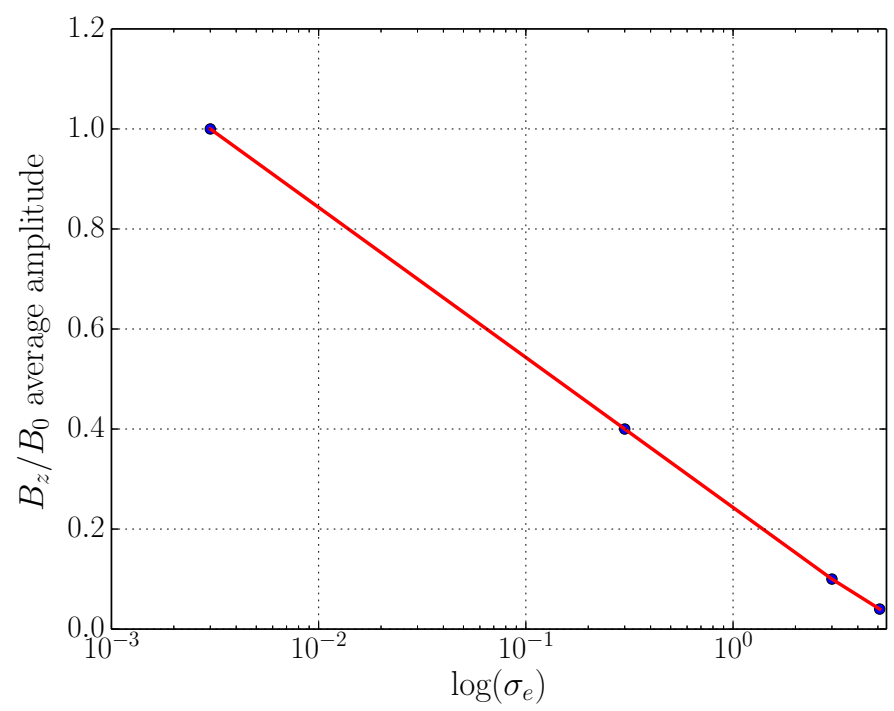

Figure 6: Average amplitude of oscillations in $B_{z} / B_{0}$ as a function of the electron magnetization $\sigma_{e}$ obtained in 2D simulations of pair plasmas. The simulations used 100 particle per cell per species and the same spatial resolution, $\lambda_{s e}=15$, as in runs with electron-ion plasmas. 
magnetization $\sigma$, electron magnetization grows linearly with the ion-to-electron mass ratio, $\sigma_{e}=$ $\left(1+m_{i} / m_{e}\right) \sigma$. For $\sigma=0.1$ assumed here and for the realistic mass ratio, electron magnetization is in the regime $\sigma_{e} \gg 1$, in which the efficiency of the precursor wave emission is much lower than in plasmas with $\sigma_{e} \lesssim 1$. We preliminarily quantified this effect by performing 2D simulations with pair plasmas for a range of electron magnetization between $\sigma_{e}=3 \times 10^{-3}$ and $\sigma_{e}=5.1$, the latter corresponding to $\sigma=0.1$ and $m_{i} / m_{e}=50$. Figure 6 shows the obtained dependence of the precursor wave amplitude on $\sigma_{e}$, demonstrating an exponential decrease of precursor wave intensity with the electron magnetization. This means that very efficient wave emission might be needed in plasma simulations with reduced mass ratios to demonstrate electron heating and significant electron-ion coupling in electron-proton plasmas. However, multi-dimensional effects may also play a significant role, as expected for high Lorentz factor shocks [12]. High-resolution 2D simulations of mildly-relativistic electron-ion shocks are thus required to properly examine the shock physics under blazar conditions. This will be a subject of our future research.

\section{Acknowledgments}

The work of A.L. and J.N. is supported by Narodowe Centrum Nauki through research project DEC-2013/10/E/ST9/00662. M.P. acknowledges support through grants PO 1508/1-1 and PO 1508/1-2 of the Deutsche Forschungsgemeinschaft. Numerical simulations have been performed on the Prometheus system at ACC Cyfronet AGH.

\section{References}

[1] M. Sikora, et al., ApJ 779 (2013) 68

[2] D. Alsop and J. Aarons Phys. Fluids 31 (4) (1988)839-847

[3] L. Sironi and A. Spitkovsky, ApJ 698 (2009) 1523-1549

[4] M. Hoshino and J. Aarons, Phys. Fluids B 3 (1991) 818-833

[5] Y. Lyubarsky, ApJ 652 (2) (2006) 1297

[6] Y. A. Gallant, et al., ApJ 391 (1992) 73-101

[7] M. Hoshino, et al., ApJ 390 (1992) 454-479

[8] A. Spitkowsky, in AIP Conference Series 801 (2005) 345-350

[9] M. Hoshino, ApJ 672 (2008) 940-956

[10] A.B. Langdon, et al., Phys. Rew. Lett. 61 (1988) 779

[11] E. Amato and J. Aarons, ApJ 653 (2006) 325

[12] M. Iwamoto, et al., ApJ 840 (2017) 52

[13] L. Sironi and A. Spitkovsky, ApJ 726 (2011) 75

[14] A. Stockem, et al., ApJ 755 (2012) 68

[15] J.Niemiec, et al., ApJ 684 (2008) 1174-1189 